\title{
Relapse of Graves' Disease and Severe Thyroid Eye Disease following Total Thyroidectomy.
}

Isuri Kurera, Arshia Panahloo

Thomas Addison Unit

St Georges Hospital, London

Total thyroidectomy is one of the definitive treatments for Graves' disease. This case describes the rare recurrence of thyroid eye disease (TED) and thyrotoxicosis due to thyroid remnant tissue.

We present a 58 year old lady with Graves' disease first seen in 2005 with positive TSH receptor antibodies (TSHRab) level of 2.5 U/L. She had a large multinodular goitre at presentation and this continued to grow with retrosternal extension and subsequent tracheal deviation. She underwent total thyroidectomy in 2014. At this point she had mild TED treated with lubricant eye drops. She had stable thyroid functions on levothyroxine (125 micrograms od).

Two years later she developed sudden thyrotoxicosis and her thyroxine replacement was stopped. Her thyrotoxicosis persisted and she developed worsening TED needing ophthalmology input. Her TSHRab levels were now high at $26.4 \mathrm{U} / \mathrm{L}$. Technetium (Tc) thyroid uptake scan demonstrated mediastinal thyroid remnant low in the thoracic cavity. Her thyroid remnant would have required thoracotomy for removal. She was commenced on Carbimazole titration therapy with continued ophthalmology input. Her latest thyroid functions are normal on Carbimazole with stable eye disease. She is now considering radioactive iodine therapy under steroid cover as definitive therapy for her Graves' disease.
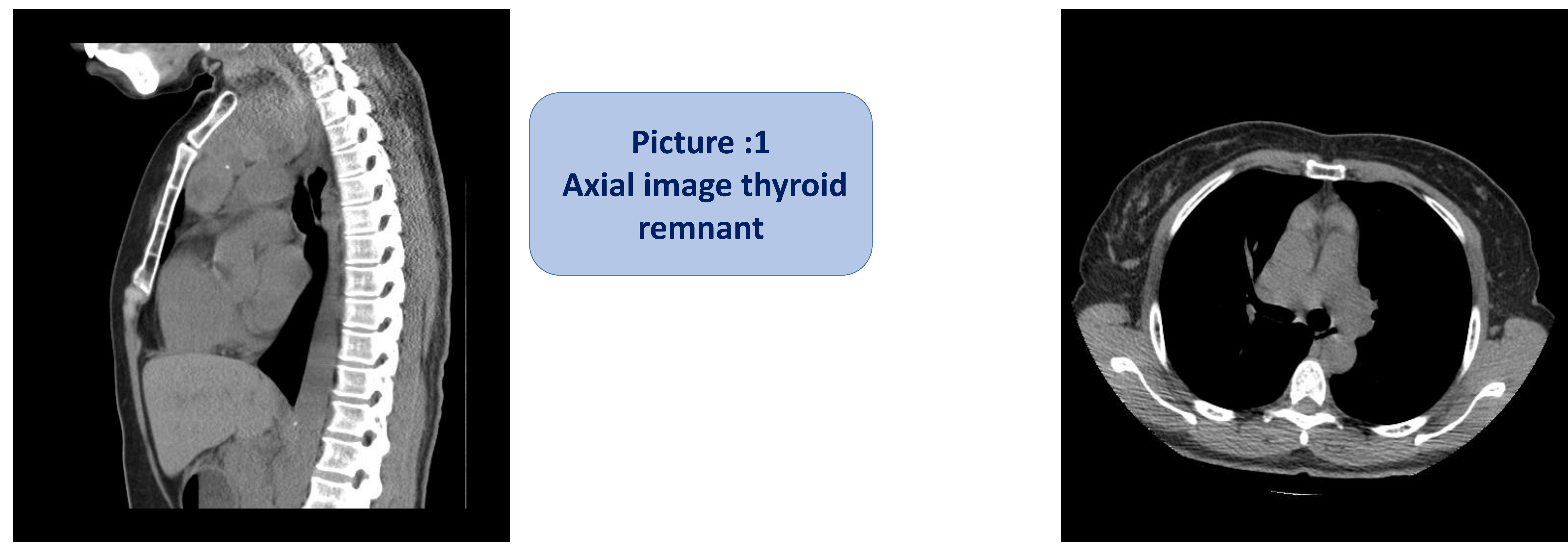

Picture: 2

Picture :1

Axial image thyroid remnant

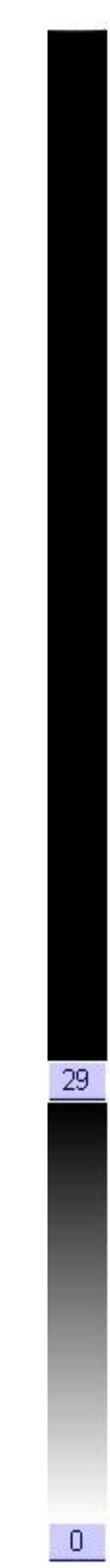

RT

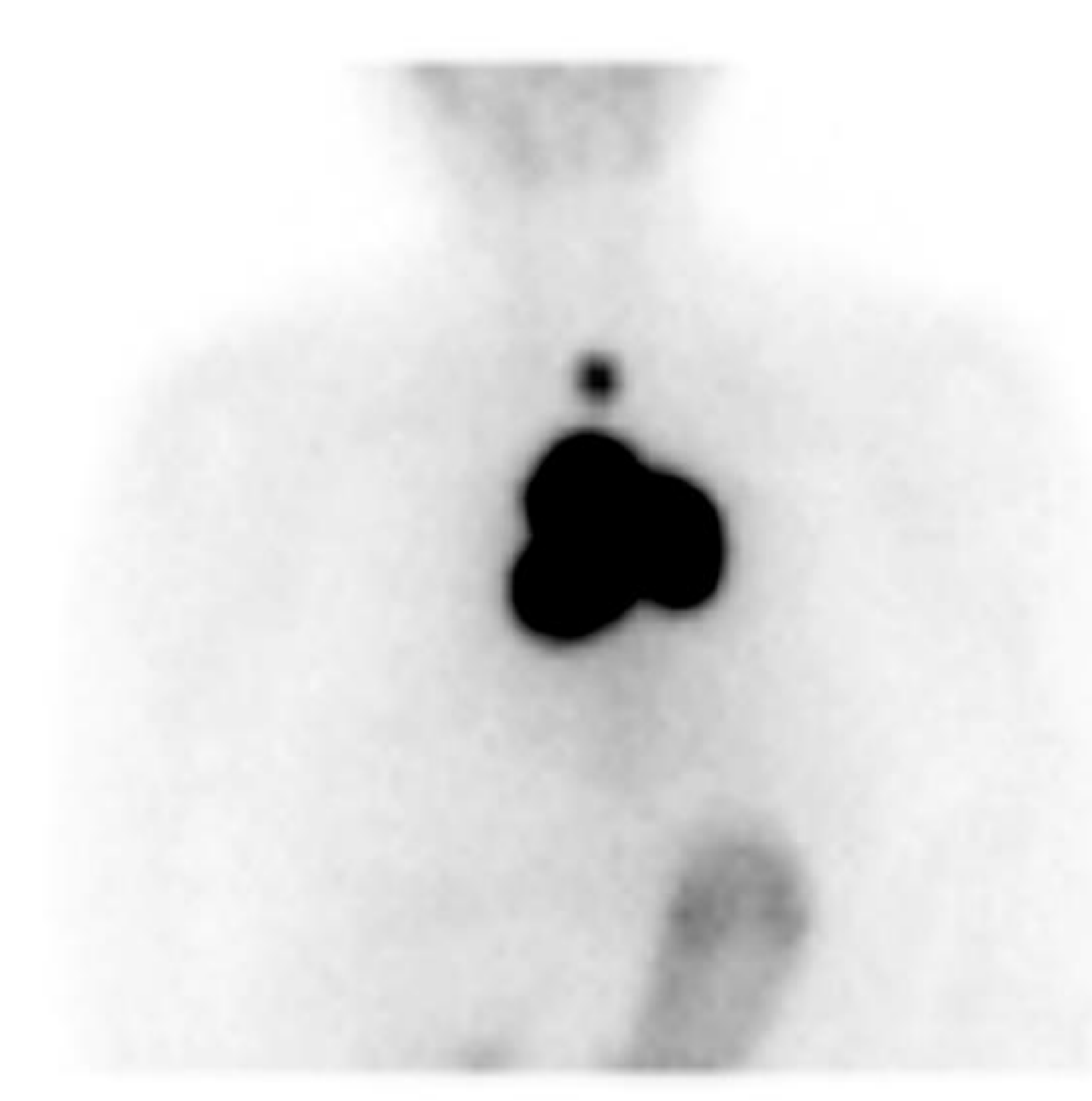

ANTERIOR UNZOOMED 1175K Coun!

Image 3: Thyroid

uptake scan 1
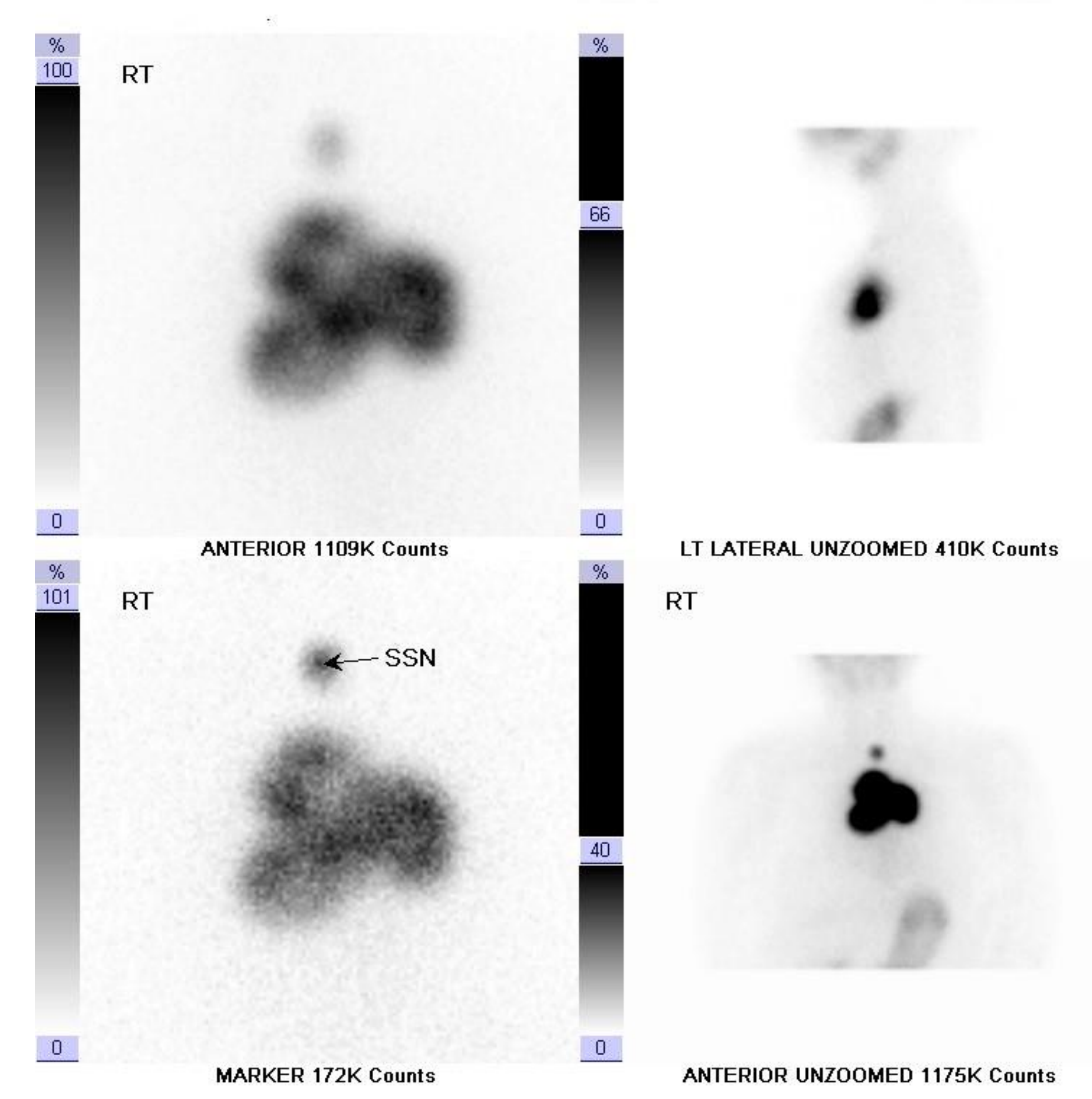

임

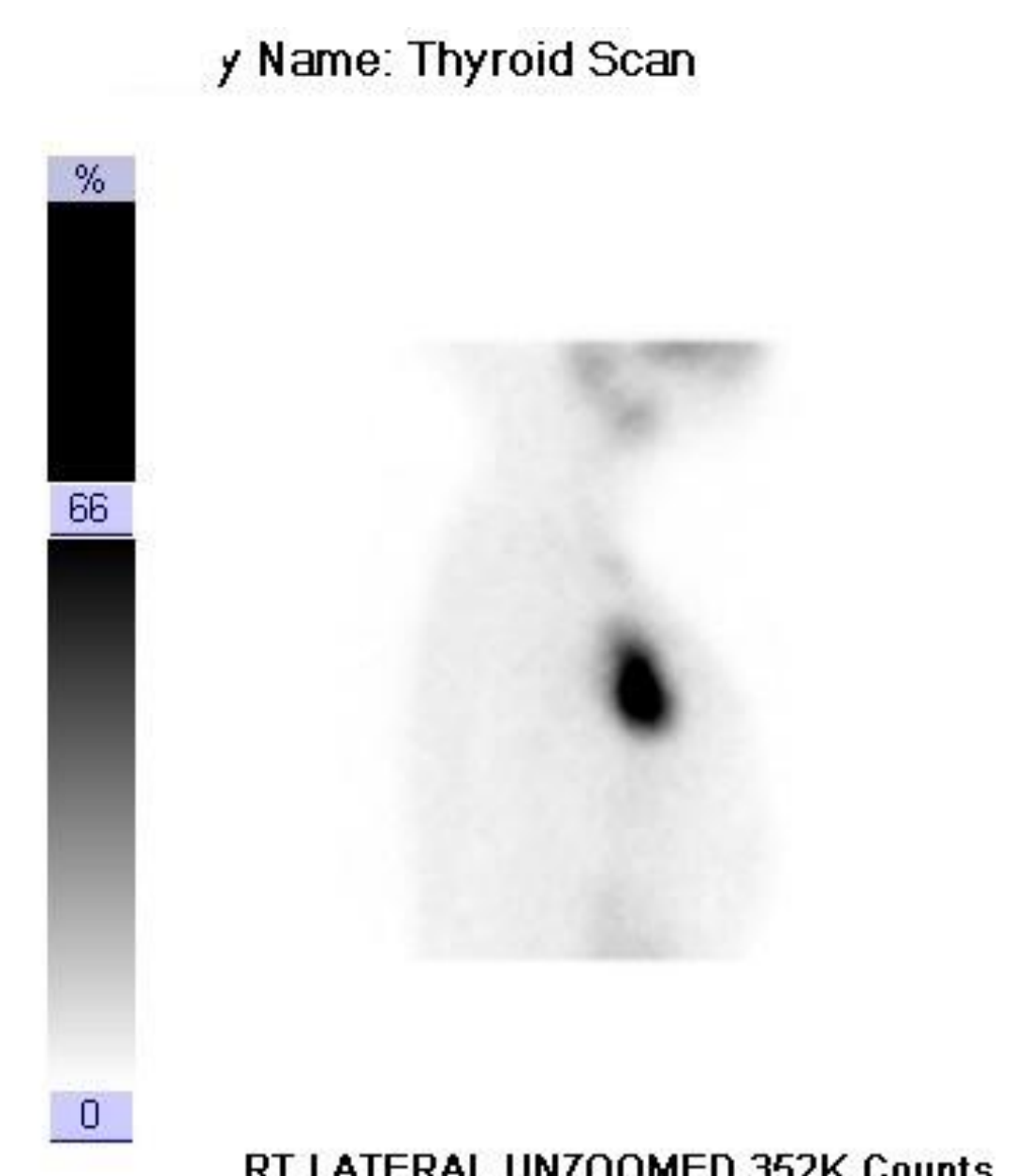

Sagittal image of thyroid remnant 